\title{
Non-Invasive Measurement of Various Health Parameter through
} IOT

\author{
${ }^{{ }^{1}}$ Gauri Deshmukh, ${ }^{2}$ Tanuja Dhope, ${ }^{3}$ Munmun Ghosal, ${ }^{4}$ K.K.Paliwal \\ ${ }^{1,3,4}$ G.H. Raisoni College of Engineering \& Management, Wagholi, Pune \\ ${ }^{2}$ BVUCOE, Pune \\ Email:1deshmukhgauri2016@gmail.com, ${ }^{2}$ tanuja_dhope@yahoo.com \\ 3munmun.ghosal@raisoni.net, ${ }^{4}$ krishan.paliwal@raisoni.net
}

Received: $13^{\text {th }}$ December 2018, Accepted: $13^{\text {th }}$ February 2019, Published: 30 $^{\text {th }}$ June $^{2019}$

\begin{abstract}
Healthcare industry has constantly been on the forefront within the adoption and utilization of data and communication technologies for the economic aid healthcare administration and treatment. Development of Internet of Things (loT) have spread out new avenues for analysis and exploration within the all fields together with medical and health care trade. Hospitals have started victimization the cell instruments for communication intent and for this intent loT has been used and joined with Wi-Fi sensing element node together with some hardware devices. The usage of a cellular agent in healthcare procedure beneath Wi-Fi community settling offers an opportunity to explore improved services for patients and staffs paying homage to medical professionals and nurses on condition that of its quality.
\end{abstract}

Keywords

Human Body Sensor; IOT; e-Health Care, Raspberry Pi

\section{Introduction}

At the present in country zone the majority of the people groups does not gets fitting way to deal with wellbeing observing and centers. So it is important to plan the viable wellbeing observing framework. A little remote gadget is goals attach with loT can shape a possible method to direct patients indirectly as opposed to dating the genuine center. The abnormal modest transducers are relocate into the human to total subtle elements through which framework get human wellness information security and for investigation for treatment. The gathered information is then send to remote station by means of dissimilar correspondence innovations empowered Wi-Fi connect with global net. From information originated from web the medicinal experts can seize end and subsequently outfit benefits halfway. Primary favorable position of this electronic human services is it gives a great relaxation to sufferers and social insurance supporters, and furthermore upgrades the top of the line demonstrating presence. Wellbeing is one of the worldwide issues for humankind. As indicated by the Parts of the World Health Organization (WHO), the greatest possible wellbeing principles are important rights of people. Sound persons help the weight on doctor's facilities, centers and medicinal services specialists, lessening the weight on open wellbeing systems, altruistic associations, and government or non-administrative associations. With the end goal to keep an individual solid by using present day human services framework, it is important to have a best in class medicinal framework that can be gotten to adequately and effortlessly.

A modernized therapeutic framework must give better restorative administrations whenever, anyplace, in a more efficient and an easy to use shape. As of now, medicinal frameworks are encountering social changes from conventional ways to deal with modernized patient-driven methodologies. In conventional methodologies, human services labourers assume a noteworthy job. They have to meet the patient for the fundamental determination. This methodology has two main issues. At first, medicinal staff should dependably be close to the patient constantly and furthermore patients are associated with bedside biomedical gear for a specific timeframe while they are hospitalized. With the end goal to tackle these two issues, different patient-arranged strategies had been proposed. In this methodology, quiet data assumes an essential job in the conclusion and anticipation of infections. A vital component of this methodology is a dependable and promptly accessible patient observing framework (PMS). The requirement for constant chronicle and warning of patient's imperative signs is most vital for viable PMS. By applying the rewards of present day bio instrumentation, PCs, and media communications advancements, current patient observing system require a stored data, to show, and transfer physical information from patient's body whenever.

\section{Problem Statement}

In many hospitals, examination is completed so as to gather the data of patient's condition. Continuous and frequent observing of patients is needed supported their health state. This results in drawbacks like Timeconsuming measurement, Low monitor accuracy, Difficulty in automatic monitoring patient. To remove all such drawback current Body sensor network has been implementing which provides a fully automated and wireless patient body monitoring. 


\section{Backgrounds and Related Work}

D Sarfraz Fayaz Khan states in his paper that for the distinguishing proof of gadget and data preparing of gear the RFID, WSN, and so on are utilized. Body zone organize (BAN) will contribute a huge obligation in moving broad extent of offers along these lines BAN machines being practiced inside the region or embed in interior body. However, the present gadgets wellbeing frameworks don't utilize mobiles, laptops to transfer basic information identified with the patients' health. In this projected framework I have propose the data of a humans wellbeing to the therapeutic experts by means of advanced mobile phones utilizing IoT. This methodology will uprightly administer the anatomical contentions of the circumstances and some varieties in the predetermined factors will activate cautions transfer to the restorative expert. The relationship of the WEAN with Android based phone progresses a huge common sense. In this manner hardware medicinal service has the ability of overall acknowledgment.[1]

Deepesh K Rathore and et al study the doctor's facilities and patient's consideration unit and thusly suggest a framework that can be extremely valuable for healthcare use. In this specialists can screen the patients state from where they are sitting and gives quick service to the patient. I can be beneficial in checking passings because of postponement in convenient consideration. Further if there should arise an occurrence of crisis, the specialist is likewise educated about the patient by means of SMS, in this manner even in absence of specialist, he will be instantly educated about patients' situation. It helps the patients, even in crisis, they receive quick cure. [2]

Ajinkya and et al infer that by and large in basic case patients should be checked ceaselessly for their pulse, oxygen immersion level, circulatory strain, temperature, beat rate and cardiac and so on. In old techniques, specialists should be available physically without hesitation, so the constant wellbeing checking framework is used each field, for example, healing facility, home consideration unit, sports utilizing remote sensor arrange. This wellbeing observing framework use for annal ailments patients who have day by day registration. Thus, specialists structure a framework as versatile gadget. Analyst planned distinctive wellbeing observing framework dependent on necessity. Diverse stage similar to controller, PIC controller and inserted frameworks are utilized to structure the framework dependent on this execution and in the ongoing years cloud based e-medicinal services frameworks have developed. In future this framework will be constructed on FPGA. [3]

Creator of this paper examined the execution of the remote Health checking framework must be expanded with the goal that Patient Data can be transmitted safely and effectively, where the prime parts are Packet Loss Ratio and Energy utilization. The biomedical remote sensors work at a cycle with low vitality utilization for expanding the future of the system. Though from the outcomes it tends to be surmised that the Packet Loss Ratio and Energy Consumption is little for LEACH Protocol [4]

Neha and et al reasoned as paper gives or projected another IoT founded patient's wellbeing factor recording framework which can be available by the doctors of an association. The framework works by detecting the heartbeat rate, temperature of the patient and continually spares the refreshed information to the server of the framework. The given framework is projected with the end goal to decrease the weight of patients when they call to the doctor however concerned doctor can't go to the patient, consequently persistent need to move to another doctor then the different doctor starts dealing with patient by getting to the associated information from server. [5]

The present doctor's facility driven medicinal services framework is getting to be wasteful to treat conditions that request prompt treatment, for example, heart strokes. Along these lines, the center is currently tilting from healing facility driven treatment to tolerant driven treatment. Undertaking in [6] proposes a wellbeing checking framework which screens essential parameters of the patient, for example, body temperature and pulse utilizing sensors which are associated with a raspberry pi kit. Undertaking includes alarming specialist by sending SMS if some essential factors of patient goes amiss from ordinary esteem. Apart from serving the specialist screen the patient's fundamental wellbeing factors the given wellbeing observing framework additionally guarantees that patient takes the recommended drug at the correct occasions. The raspberry pi goes about an individual server which records the subtle elements of the patient's medicine. Patient gives suggestions to catch meds using SMS as indicated by patients medicine.

As medicinal services administrations are imperative piece of our general public, mechanizing these administrations diminish the weight on people and facilitates the estimating procedure. Additionally the straightforwardness of this framework causes patients to confide in it. At the point when edge esteem is achieved, the caution framework that comprises of ringer and LED alarms the specialists and he can act all the more rapidly. The target of creating checking frameworks is to lessen medicinal services costs by diminishing doctor calls, hospitalizations, and indicative checking system. GSM innovation causes server to refresh the patient information on site. Many enhancements created in this framework for improving it, for example, including further developed devices. Biological data of patient is distributed wirelessly. It can given to researchers of restorative fields to break down esteem and discover designs or for other research work. [7]

There are a few spots of Internet of Things (IOT) is utilized. For example, savvy condition, keen home, shrewd city, brilliant stopping, horticulture fields and medicinal fields. In medicinal field likewise, there are a few procedure are utilized web. In [8], screen patient's temperature, pulse rate and developments utilizing Raspberry 
$\mathrm{Pi}$. In the wake of associating net to the Raspberry Pi board it go about as a server. At that point server is naturally transfer information to webserver. Then these parameters are screen utilizing website page anyplace on the planet utilizing PCs, advanced mobile phone and so on. In the event that these parameters are goes to anomalous, it will consequently sends ready message to the specialist.

As we realize that it is exceptionally difficult to monitor the patient for 24 hour. Therefore the condition of patient well being i.e, respiration rate, Body Temperature, and ECG. These parameters can be estimated by using a few sensors. The gathered information through the sensors is then exchanged to the web. What's more, Via web this information is exchanged to PCs which are enrolled to the server of the database and the cell phone of the specialists. In the wake of breaking down the information specialists would then be able to recommend the prescription dependent on the information results appeared by the framework. Displayed model will limit the weight on patients to visit the specialists each time for checking of these well being parameters. [9]

A remote social insurance checking framework by methods for utilizing cell phones and sensors can be executed in a worldwide system with the assistance of Arduino and Raspberry Pi. The gadgets and IoT assembles and share data with one another, making it conceivable to gather, examine and screen information all the more precisely. In this way IoT can be utilized for observing the patient and giving administrations in an opportune way. The proposed framework [10] can be improved and stretched out by utilizing other intrusive and in addition nonobtrusive sensors for grabbing fundamental therapeutic possibilities of a patient. This can be additionally investigated, put away and exchanged on a worldwide stage. Mega Arduino can likewise be utilized that is equipped for interfacing numerous sensors in the meantime. This will help indicating results parallely with the goal that simplicity of association and efficient can be encouraged.

\section{Proposed System}

A smart healthcare system has temperature, respiration; patient's body movements and cardiac reading is observed with the help of Raspberry Pi. The given sensors data transfer to Raspberry Pi through amplifier circuit and signal conditioning unit (SCU), as signal levels are low (gain), hence amplifier is essential for increasing signal gain and transfer the signals to the Raspberry Pi. Raspberry pi based on Linux operating system. It works as a small processor. In this system patients body temperature, body movements, respiration and cardiac rate is measured using corresponding sensors and observed on the computer screen using Raspberry Pi. It can also observed anywhere in the world using internet source.

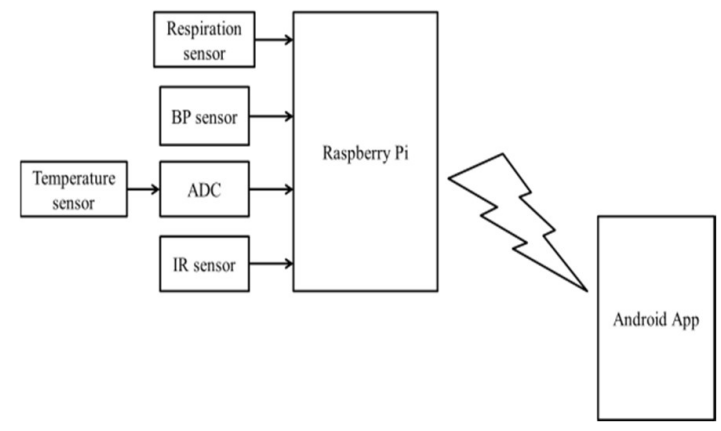

Figure 1: Projected System

The projected technique of patient observing system is monitor patient's body temperature, Blood pressure, and Respiration rate and body movements using Raspberry Pi. once connecting net to the Raspberry Pi it act as a server. Then the server is mechanically sends records to the website. By Internet Protocol address anybody will monitor the patient's health position anyplace within the world using PCs, tablets and cell phones. If these factors are falls to irregular it will spontaneously shows aware mail to the specialists and families. After full hardware completion process, then putty software is used for completion of full project Then MIT app inventor software is used for transfer these parameters from Raspberry Pi to Android App.

This paper proposes a period low value electronic saline observing and system which may mechanically monitor the saline flow rate, remaining time and might conjointly management infusion rate. It can wirelessly send the data to server and show the ends up in the form of saline driblet rate, failure condition, remaining time to empty the saline bottle and show infusion volume displayed on central monitor The important concept of this projected system is to supply value effective, reliable, comfy, convenient, easy and automatic saline flow observing and control system that interface show of bottle finished standing is effortlessly enforced in any hospital and might be straight forward for nurses to observe the saline flow and can management infusion drip rate from a distance. 


\section{Conclusion}

The essential factors of the patient body such as humidity, temperature and respiration level are checked by the respective sensors. The measured parameters are transmitted to the cloud through the Wi-Fi module. Finally we can see the analysis of patient body parameter on mobile phone user as well as laptop user through browser as well as physician get the information about patient body parameter through mail. Only authorized person has access to this data.

\section{References}

[1] Health Care Monitoring System in Internet of Things (loT) by Using RFID Sarfraz Fayaz Khan 20 17 IEEE

[2] Wireless Patient Health Monitoring System Deepesh K Rathore, Ankita Upmanyu, Deepanshu lulla

[3] Mr. Ajinkya A. Bandegiri Dr. Pradip C. Bhaskar Real-Time Health Monitoring System: A Review International Journal of Trend in Scientific Research and Development (IJTSRD) ISSN: 2456-6470 Volume - 2 | Issue - 1 | Nov-Dec 2017

[4] Patient Health Monitoring in Wireless Sensor Network, Antima khatod, Sandhya Sharma Volume 2, Issue 02, February- 2017 International Journal Of Innovative Science And Research Technology ISSN No:-2456 -2165 IJISRT17FB07 www.ijisrt.com 24,

[5] Neha, Poonam Kumari2, H.P.S Kang SMART HEALTH MONITORING SYSTEM International Journal of Recent Trends in Engineering \& Research (IJRTER) Volume 03, Issue 06; June - 2017 [ISSN: 2455-1457]

[6] Shreya Rajkumar Malavika Srikanth Dr. N. Ramasubramanian "Health Monitoring System using Raspberry PI", 2017 International Conference on Big Data, IoT and Data Science (BID) Vishwakarma Institute of Technology, Pune, Dec 20-22, 2017

[7] Nagarjuna Reddy A, G Hari Krishnan, and Raghuram D Research Journal of Pharmaceutical, Biological and Chemical Sciences Real Time Patient Health Monitoring Using Raspberry PI.

[8] R.Kumar Dr. M. Pallikonda Rajasekaran "Raspberry Pi Based Patient Health Status Observing Method Using Internet Of Things" International Conference on Current Research in Engineering Science and Technology (ICCREST-2016)

[9] Archit Sharma, 2Ruqaiya Khanam, 3Akriti Kumari, 4 Subham Singh, "A Smart Patient Health Monitoring System Using Raspberry Pi 3” October 2017, Volume 4, Issue 10 JETIR (ISSN-23495162) JETIR1710085 Journal of Emerging Technologies and Innovative Research (JETIR) www.jetir.org 507

[10] Kartikee Uplenchwar, Aditi Vedalankar "IoT Based Health Monitoring System using Raspberry Pi and Arduino" International Journal of Innovative Research in Computer and Communication Engineering Vol. 5, Issue 12, December 2017 